\title{
Transformation-mismatch plasticity in sub-millimeter iron wires
}

\author{
John D. Marvin, David C. Dunand* \\ Department of Materials Science and Engineering, Northwestern University, Evanston, IL 60208, USA
}

\begin{abstract}
Wires of $99.5 \%$ pure iron with 406 and $508 \mu \mathrm{m}$ diameter were subjected to a uniaxial tensile stress while being thermally cycled about the $\alpha-\gamma$ allotropic transformation temperature. The strain increments per cycle are proportional to the applied stress in the range 1-22 MPa, indicating that transformation-mismatch plasticity is the dominant deformation mechanism. The strain increments for the wires have the same magnitude as those reported for bulk iron samples, thus, indicating that the internal mismatch strains responsible for this deformation mechanism are undiminished in the wires, despite their high surface-to-volume ratio. Very high average strain rates (up to $3 \times 10^{-3} \mathrm{~s}^{-1}$ ) were achieved through resistive heating and convective/radiative cooling of the thinnest wires.
\end{abstract}

(C) 2005 Elsevier B.V. All rights reserved.

Keywords: Allotropic transformation; Plasticity; Superplasticity; Creep; Iron; MEMS

\section{Introduction}

Phase transformations can induce plasticity in iron and steels through two distinct mechanisms: (i) preferential selection by the applied stress of martensite variants with a non-zero shear [1-4] or (ii) biasing by the applied stress of internal stresses produced by the volumetric mismatch between allotropic phases during the transformation [5-7]. For the former mechanism, which is often called transformation-induced plasticity (TRIP), the mechanism responsible for deformation is exhausted when all martensite variants are aligned, so that large strains (greater than $\sim 10 \%$ ) cannot be accumulated. There is no such limitation for the latter mechanism, transformation-mismatch plasticity (TMP), as internal mismatch stresses are generated at each crossing of the allotropic range, so that strain increments can be accumulated to very large total strain values $(>100 \%)$ upon repeated thermal cycling (previous experiments in iron and steels are summarized in Ref. [6]). That mechanism is then also called transformation superplasticity and has been demonstrated in many allotropic metals, alloys and composites $[5,8,9]$.

To date, all investigations of transformation-mismatch plasticity have studied bulk samples, where the smallest dimension is larger than $1 \mathrm{~mm}$, with potential applications for forming bulk objects [10,11]. Recently, micro-forming of objects in the sub-millimeter range has become of interest to shape microelectro-mechanical systems (MEMS) parts [12], as demon-

\footnotetext{
* Corresponding author. Tel.: +1 847491 5370; fax: +1 8474676573

E-mail address: dunand@northwestern.edu (D.C. Dunand).
}

strated recently with a superplastic $\mathrm{Zn}-\mathrm{Al}$ alloy superplastically extruded into gears with $50 \mu \mathrm{m}$ diameter [13]. In that work where superplastic deformation occurs by sliding of grains less than $10 \mu \mathrm{m}$ in size, the sample dimensions were larger than the grain size, so that no effect of sample size was expected upon the deformation. By contrast, transformation superplasticity relies on the creation of internal stresses within the material, which may be affected by sample size; as the surface-to-volume ratio increases, an increasing fraction of the material volume is in the vicinity of the free surface, where internal stresses are reduced. Another important parameter that is affected by the surface-to-volume ratio is the cooling rate achievable under convective conditions (and to a lesser extent the heating rate); the faster the heating and cooling rates are, the higher the cycling frequency can be, and thus, the higher the average strain rate for the material being formed.

The present paper reports on a first investigation of transformation-mismatch plasticity for wires with sub-millimeter diameter. We utilize here iron because of its well characterized behavior under TMP conditions and its lack of sensitivity with respect to contamination, but our conclusions are expected to apply to steels and other metals exhibiting transformationmismatch plasticity and superplasticity, e.g., Co [5,14], Ti [5,15], $\mathrm{Zr}[5,16]$ and their alloys [17].

\section{Experimental procedures}

Wires of $99.5 \%$ pure iron with diameters of 406 and $508 \mu \mathrm{m}$ were purchased from ESPI Corp. (Ashland, OR). A chemical 


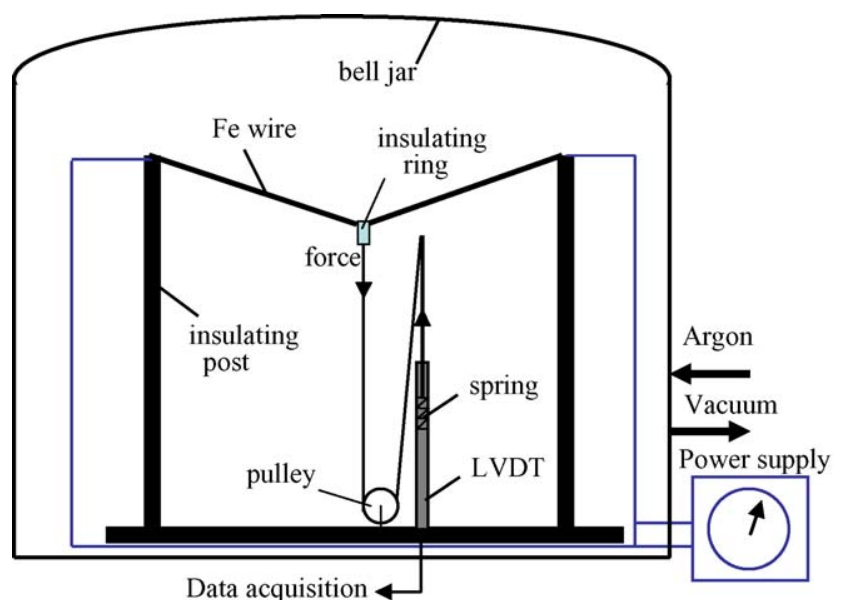

Fig. 1. Schematic of apparatus used to subject wires to rapid thermal cycling under an applied tensile stress.

analysis by the supplier provided the following composition (in wt. \%): $0.33 \% \mathrm{Mn}, 0.053 \% \mathrm{Sn}, 0.045 \% \mathrm{Cu}, 0.030 \% \mathrm{Cr}, 0.017 \%$ $\mathrm{Si}, 0.0065 \% \mathrm{Ni}, 0.0063 \% \mathrm{Al}$, balance Fe. Given the overall purity of $99.5 \%$, a carbon content below $0.013 \%$ is inferred.

An apparatus, shown schematically in Fig. 1, was built to subject these wires to rapid thermal cycling under an applied tensile stress and with cover gas. The wire is fixed between two insulating vertical posts connected to a power supply, allowing for rapid resistive heating. Cooling occurs by radiation and convection in argon. The wire is subjected at its middle point to a vertical force from a spring-loaded linear voltage displacement transducer (LVDT), connected to the iron wire through an insulated ceramic ring and a thin nylon string. Voltage is varied with square cycles for transformation plasticity experiments or kept constant for creep experiments. The vertical motion of the wire mid-point is recorded continuously through the LVDT, whose spring is also providing the vertical force on the wire; additional weights are used for high-stress experiments. The strain is calculated from the wire mid-point vertical deflection, assuming a uniform stretching of the wire as a result of plastic deformation over the heated length of the wire (discounting the cold ends at the posts and the small cold region in contact with the ceramic ring). The tensile stress is calculated as a function of vertical deflection by taking into account the decreasing force from the spring, the decreasing angle of the wire with respect to that force and the decreasing wire cross-section, which is calculated under the assumption of uniform thinning over the heated length.

\section{Results}

Fig. 2 is a plot of strain versus time for creep experiments conducted for a series of increasing voltages (and thus, temperatures) on a $508 \mu \mathrm{m}$ wire under a roughly constant tensile stress of $9 \mathrm{MPa}$ (decreasing from 10.4 to $8.2 \mathrm{MPa}$ from the beginning to the end of the experiment). After each step increase in temperature, a rapid extension occurs due to thermal expansion. Only a small amount of primary creep is observed before the establishment of secondary creep. As expected, the creep rate increases

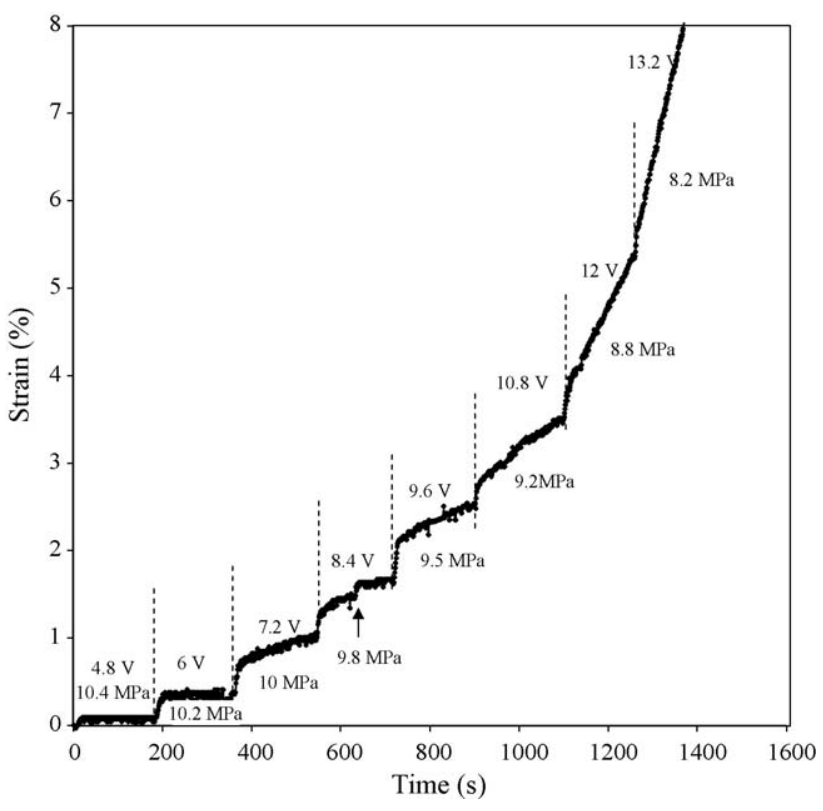

Fig. 2. Plot of strain vs. time for a $508 \mu \mathrm{m}$ wire deformed for a series of increasing voltages (and thus, temperatures) under a near-constant stress (values of voltage and stress are given for each creep segment). Transformation occurred at the time marked with an arrow.

with increasing temperature. The $\alpha-\gamma$ transformation occurred during one of the creep period and is associated with a small TMP strain increment, marked with an arrow in Fig. 2. This occurred probably as a result of the steady elongation and thinning of the wire that increases its resistance, and thus, its temperature, at constant applied voltage. As expected from the higher creep resistance of the close-packed austenite phase, the creep rate of $\gamma$-Fe after the phase transformation $\left(7 \times 10^{-6} \mathrm{~s}^{-1}\right)$ is lower than that of $\alpha$-Fe before the transformation $\left(2.5 \times 10^{-5} \mathrm{~s}^{-1}\right)$; the difference in rates is, however, less than predicted in Ref. [18].

Fig. 3 is a plot of strain versus time for three experiments conducted until fracture. A first curve is for a TMP experiment performed on a $406 \mu \mathrm{m}$ wire with square voltage cycles $(0-12 \mathrm{~V})$ with $3 \mathrm{~s}$ period, for which the average stress decreased near linearly with time from 10 to $\sim 0 \mathrm{MPa}$; necking was observed at high strains, so that the stress was higher at necks. A second curve is for a creep experiment performed on a $508 \mu \mathrm{m}$ wire near the maximum temperature in the $\gamma$-phase (constant voltage of $12 \mathrm{~V}$ ) under a near-constant stress of 5.9 MPa. A third curve shows thermal cycling in the $\gamma$-phase for a $508 \mu \mathrm{m}$ wire (square 9.6-12 $\mathrm{V}$ cycles with $3 \mathrm{~s}$ period) under a near-constant stress of 8.6 MPa, performed to explore the effect of thermal mismatch stresses without phase transformation. It is apparent that the TMP experiment results in average strain rate and fracture strain that are much higher than for both creep control experiments, despite the higher stress used for the latter experiments. Fig. 3 is, thus, direct confirmation that transformation-mismatch plasticity is the dominant deformation mechanism in the wires when thermally cycled about their phase transformation temperature. Grain size measured on a cycled wire was $>40 \mu \mathrm{m}$, thus, ruling out micro-structural superplasticity as a deformation mechanism. 


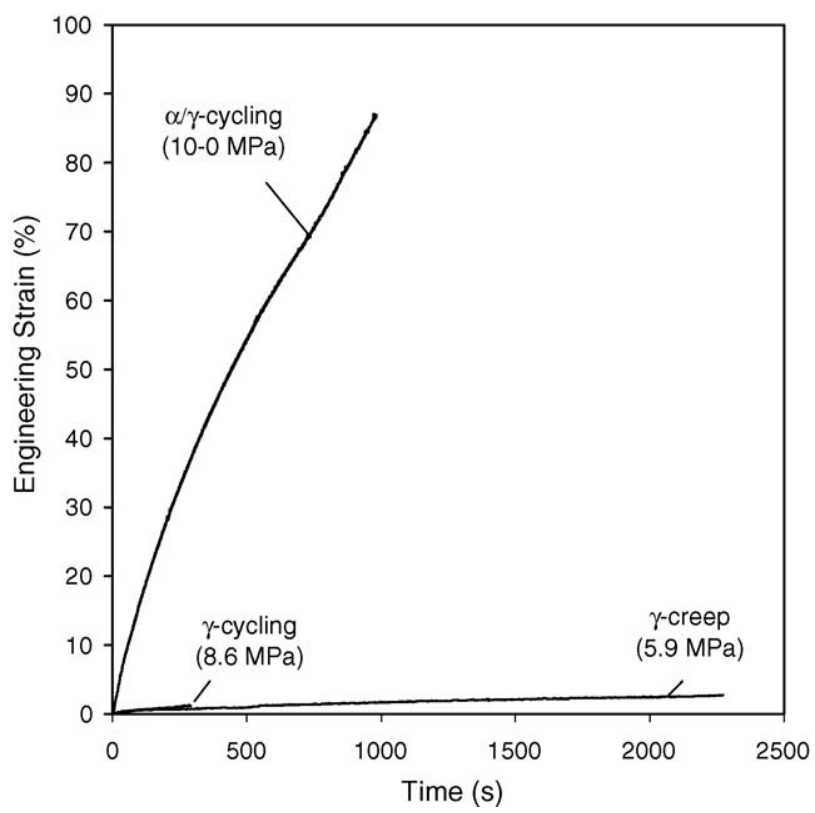

Fig. 3. Plot of strain vs. time for wires deformed to fracture: $406 \mu \mathrm{m}$ wire deformed under thermal allotropic cycling with square $0-12 \mathrm{~V}$ cycles with $3 \mathrm{~s}$ period under a stress decreasing near linearly with time from 10 to $\sim 0 \mathrm{MPa}$; $508 \mu \mathrm{m}$ wire deformed under isothermal creep at constant stress (5.9 MPa) and temperature in the $\gamma$-phase (using a $12 \mathrm{~V}$ voltage); $508 \mu \mathrm{m}$ wire deformed under constant stress $(8.6 \mathrm{MPa})$ under thermal cycling conditions in the $\gamma$-phase (square $10-12 \mathrm{~V}$ cycles) without phase transformation.

Fig. 4 shows a plot of strain versus time for a $508 \mu \mathrm{m}$ wire subjected to a series of allotropic cycles with period increasing from 4 to $60 \mathrm{~s}$, under a near-constant stress of $6.5 \pm 1 \mathrm{MPa}$ (decreasing from 7.5 to $5.6 \mathrm{MPa}$ ). Also, shown is the creep curve plotted in Fig. 3 (temperature: upper cycle value, stress: 5.9 MPa), which again illustrates the large increase in deformation rates achieved through transformation-mismatch plasticity. However, as the cycling period increases, the average strain rate of the segment decreases, as expected from the reduced frequency of transformations. Fig. 5 shows, for the experiment reported in Fig. 4, the

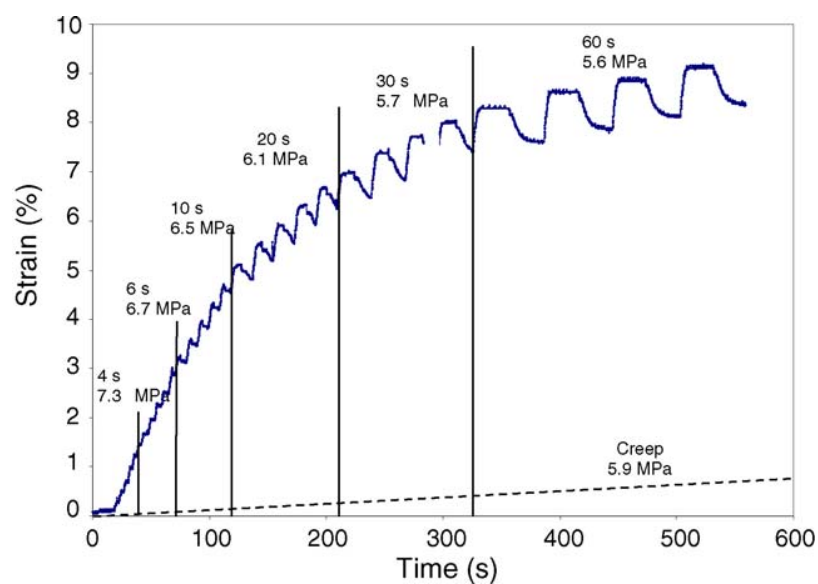

Fig. 4. Plot of strain vs. time for a $508 \mu \mathrm{m}$ wire deformed by allotropic cycling, with cycle period increasing from 4 to $60 \mathrm{~s}$. Period and stress are given for each segment.

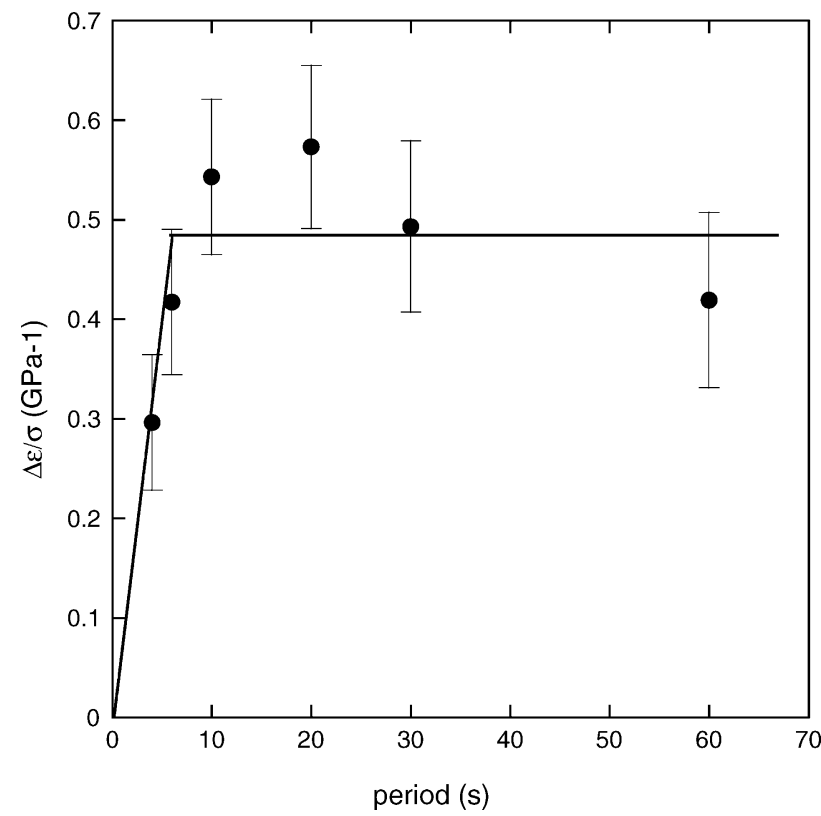

Fig. 5. Plot of strain increment per cycle (normalized by applied stress) vs. cycle period for the $508 \mu \mathrm{m}$ wire experiments shown in Fig. 4.

cycling period plotted against the strain per cycle normalized by the applied stress $\Delta \varepsilon / \sigma$, which is expected to be constant for TMP conditions, where the average stress exponent is unity. Fig. 5 indeed shows an approximately constant value (within experimental error), except for the shortest cycling period of $4 \mathrm{~s}$, for which $\Delta \varepsilon / \sigma$ is well below the average line. For $406 \mu \mathrm{m}$ wires, a plateau was also observed down to the shortest period of $3 \mathrm{~s}$.

Fig. 6 summarizes the stress dependence of TMP strain increments for a large number of experiments performed on both 406 and $508 \mu \mathrm{m}$ wires, using 3-10 and 6-60 s periods, respectively.

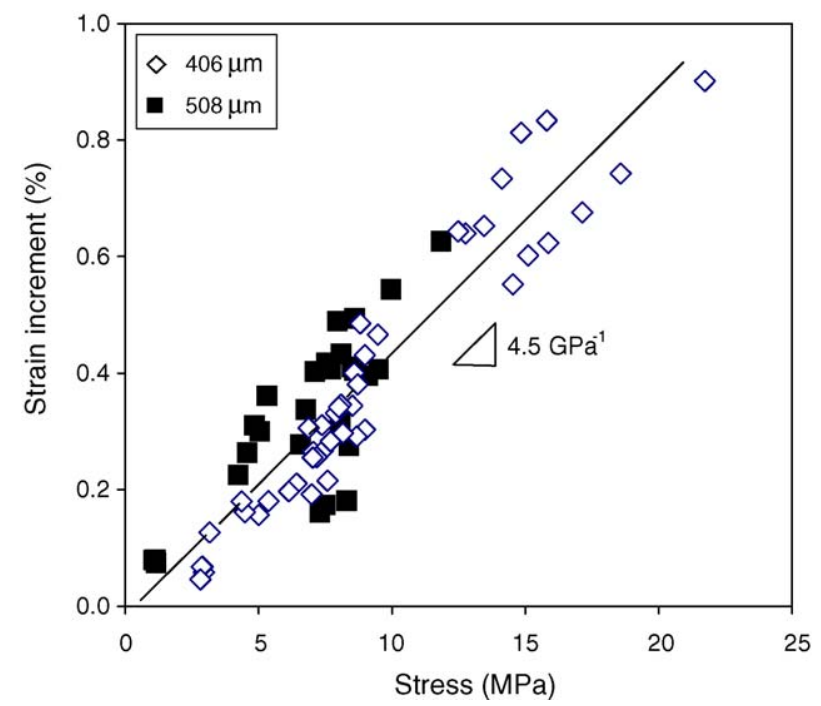

Fig. 6. Plot of strain increment per cycle vs. applied stress for $406 \mu \mathrm{m}$ wires ( $3-10 \mathrm{~s}$ periods) and $508 \mu \mathrm{m}$ wires (6-60 s periods). Error bars are not shown but are estimated at $\pm 0.05 \%$ for strain and $\pm 0.5 \mathrm{MPa}$ for stress. The best-fit line has a slope of $4.5 \mathrm{GPa}^{-1}$ and intersects the stress axis near the origin. 
The strain increments are the average of typically four cycles over which the stress was averaged. A given specimen provided three to seven data points, with the total accumulated strain never exceeding 30\%. Also shown in Fig. 6 is the best-fit line to both data sets, with a slope of $0.45 \mathrm{GPa}^{-1}$ and an intersection on the $x$-axis very close to the origin.

\section{Discussion}

Fig. 3 shows an engineering fracture strain of $87 \%$ accumulated after 328 cycles with $3 \mathrm{~s}$ period. The fracture strain for the present $406 \mu \mathrm{m}$ wire is much smaller than the strain in excess of $450 \%$ achieved for a $6 \mathrm{~mm}$ diameter samples cycled under TMP conditions with a period of $240 \mathrm{~s}$ [6]. An important factor explaining this discrepancy is the manner in which sample heating occurred. In the bulk experiments [6], radiant heating was used, so that a necked region had about the same temperature as the surrounding un-necked regions. The low strain rate sensitivity characteristic of transformationmismatch superplasticity allowed for the stable deformation of necks, resulting in high elongations to fracture. By contrast, the present wires are heated resistively, such that a neck, due to its reduced cross-section and increased resistance, is subjected to much higher temperature than the surrounding unnecked regions. Deformation then becomes highly localized at the neck, even under superplastic conditions, as studied in Ref. [17]. This localization is exacerbated for wires deforming by creep with high-stress exponents, as illustrated by the very low failure strains achieved in the creep experiments shown in Fig. 3.

For the $406 \mu \mathrm{m}$ wire experiment with $3 \mathrm{~s}$ period shown in Fig. 3, the average strain rate is $8.8 \times 10^{-4} \mathrm{~s}^{-1}$ and the initial strain rate over the initial $15 \%$ strain is $1.7 \times 10^{-3} \mathrm{~s}^{-1}$ for stresses of $9 \pm 1 \mathrm{MPa}$. An even higher value of $3.0 \times 10^{-3} \mathrm{~s}^{-1}\left(18 \% \mathrm{~min}^{-1}\right)$ was recorded for a $406 \mu \mathrm{m}$ wire subjected to a stress of $22 \mathrm{MPa}$ (highest stress point in Fig. 6). By comparison, in our previous TMP study of bulk pure iron samples with $6 \mathrm{~mm}$ diameter [6], a maximum average strain rate of $9 \times 10^{-5} \mathrm{~s}^{-1}\left(0.54 \% \mathrm{~min}^{-1}\right)$ was measured under a stress of $17 \mathrm{MPa}$ for a cycling period of $240 \mathrm{~s}$. Thus, it is apparent that the reduced magnitude (by about a factor of three) of the TMP strain increments achievable for a given stress in $99.5 \%$ pure $\mathrm{Fe}$ (present study) as compared to high purity $\mathrm{Fe}[6]$ is more than compensated by the much higher cycling rate (by a factor 80 , here) achievable through resistive heating and radiative cooling of a wire with high surface-to-volume ratio. Deformation rates in the vicinity of $10^{-2} \mathrm{~s}^{-1}$ (the value above which high-strain rate superplasticity is achieved) seem reachable in wires using a combination of higher metal purity, larger applied stresses and faster cycling rates; the latter can be achieved through improved cooling rates, e.g. by using $\mathrm{He}$ gas and thinner wires. However, as illustrated in Fig. 5, when the cycling rate reaches a critical value, there is not sufficient time during the cycle (probably, the cooling segment) for the transformation to be complete and the strain increments then decrease accordingly. This critical cycling period is about $5 \mathrm{~s}$ for the $508 \mu \mathrm{m}$ wires and less than $3 \mathrm{~s}$ for the $406 \mu \mathrm{m}$ wires.
Despite the relatively high homologous temperature of iron at the $\alpha / \gamma$ transformation temperature, transformation-mismatch plasticity of iron and steels can be modeled by assuming relaxation of internal stresses by time-independent plasticity $[5-7,19,20]$. The uniaxial strain increment $\Delta \varepsilon$ accumulated after a full allotropic transformation occurring with a superimposed uniaxial biasing tensile stress $\sigma$ is then given as [5]:

$\Delta \varepsilon=\frac{5}{3}\left|\frac{\Delta V}{V}\right| \frac{\sigma}{\sigma_{Y}}$

where $|\Delta V / V|$ is the volume mismatch between the allotropic phases $\left(|\Delta V / V|=1.1 \%\right.$ for the $\alpha-\gamma \mathrm{Fe}$ transformation) and $\sigma_{\mathrm{Y}}$ is the yield stress of the weaker phase ( $\alpha$-Fe). Eq. (1) is valid for an ideally plastic material at stresses up to half the yield stress of the weaker phase. For larger stresses and if strain hardening is present, a more complex equation can be derived $[19,20]$.

Finally, if transformation occurs with a well-defined front propagating perpendicularly to the applied stress, Schuh [21] developed a modification of Eq. (1), which predicts a near linear relationship between the strain increment and the applied stress up to an applied stress of half the yield stress of the weaker phase, but with a slope $\mathrm{d}(\Delta \varepsilon) / \mathrm{d} \sigma$ lower by a factor ca. 1.3 as compared to the value $5 / 3(\Delta V / V) / \sigma_{Y}$ given by Eq. (1). Also, a ratcheting strain of magnitude $2 / 3(\Delta V / V)=0.66 \%$ is predicted at zero applied stress [21]. The lack of any significant ratcheting strain in Fig. 6 indicates that on average, there is no net transformation front. For the present experiments, the absence of front is certainly expected on heating, which is resistive, and thus, volumetric, but a radially propagating front would be expected if cooling is solely radiative/convective through the surface. However, it is likely that small variations in wire diameters lead to transformation fronts propagating in a direction parallel to the wire on heating, thus, canceling the effect of radial fronts on cooling.

Fig. 6 shows that if Eq. (1) is fitted to the experimental data, the strain increment per unit stress is $\mathrm{d}(\Delta \varepsilon) / \mathrm{d} \sigma=0.45 \mathrm{GPa}^{-1}$ for the present $99.5 \%$ pure $\mathrm{Fe}$ wires. This value is lower than for bulk pure Fe samples which span $\mathrm{d}(\Delta \varepsilon) / \mathrm{d} \sigma=1.5-2.5 \mathrm{GPa}^{-1}$ for six investigations with iron of minimum purity of $99.95 \%$, as summarized in Ref. [6]. However, the present value $\mathrm{d}(\Delta \varepsilon) / \mathrm{d} \sigma=0.45 \mathrm{GPa}^{-1}$ is comparable to values obtained for iron of similar purity and similar very low carbon content, i.e., $\mathrm{d}(\Delta \varepsilon) / \mathrm{d} \sigma=0.42 \mathrm{GPa}^{-1}$ for $99.7 \% \mathrm{Fe}$ [22] and $\mathrm{d}(\Delta \varepsilon) / \mathrm{d} \sigma=0.32 \mathrm{GPa}^{-1}$ for $99.5 \% \mathrm{Fe}$ [23]. The yield stress calculated for the present case from Eq. (1) using the experimental value of $\mathrm{d}(\Delta \varepsilon) / \mathrm{d} \sigma$ is $41 \mathrm{MPa}$. The highest experimental stress in Fig. 6 is about half this value, so it is indeed appropriate to use Eq. (1) for all the data points.

The fact that the TMP strains for the present sub-millimeter wires are quantitatively the same as those measured on bulk samples of similar composition [22,23] indicates that transformation-mismatch plasticity is not affected by sample diameter down to $406 \mu \mathrm{m}$. This is confirmed by the lack of significant difference in stress dependence for strain increments in 406 and $508 \mu \mathrm{m}$ wires (Fig. 6). Thus, it can be concluded that the critical sample diameter, at which 
transformation-mismatch plasticity becomes less effective due to a reduction in volumetric mismatch stresses from the high surface-to-volume ratio, is below $406 \mu \mathrm{m}$. Preliminary experiments on $254 \mu \mathrm{m}$ wires (which are not reported here) indicate that the critical wire size is below this latter value. Transformation-mismatch plasticity and by extension other deformation mechanisms relying on internal stresses can, thus, be used for superplastic shaping of sub-millimeter MEMS parts.

\section{Conclusions}

- Thin iron wires (purity: 99.5\%, diameter: 406 and $508 \mu \mathrm{m}$ ) were deformed under a uniaxial tensile stress while simultaneously being subjected to thermal cycling about the $\alpha-\gamma$ transformation temperature.

- Transformation-mismatch plasticity is the dominant deformation mechanism under allotropic cycling conditions and results in much higher strain rates and failure strains than isothermal creep deformation.

- For the shortest cycling period of $3 \mathrm{~s}$ used here, an average strain rate of $3 \times 10^{-3} \mathrm{~s}^{-1}$ and a fracture strain of $87 \%$ were achieved; these values are close to those at which high-strain rate superplasticity can be claimed.

- The strain increment per cycle is proportional to the applied stress in the range $1-22 \mathrm{MPa}$, resulting in an average strain rate sensitivity exponent of unity.

- For a given stress, there is no difference in the values of the strain increments for the 406 and $508 \mu \mathrm{m}$ wires and for bulk samples reported in the literature. Thus, the high surfaceto-volume ratio in the wires does not reduce the internal mismatch stresses responsible for transformation-mismatch plasticity, which could be used for superplastic shaping of MEMS parts.

\section{Acknowledgment}

This study was supported by National Science Foundation under Grant DMR-9987593.

\section{References}

[1] J.B. Leblond, J. Devaux, J.C. Devaux, Int. J. Plastic. 5 (1989) 551-572.

[2] G.B. Olson, M. Cohen, Metall. Trans. A 6 (1975) 791-795.

[3] J.M. Diani, H. Sabar, M. Berveiller, Int. J. Eng. Sci. 33 (1995) 1921-1934.

[4] R.G. Stringfellow, D.M. Parks, G.B. Olson, Acta Metall. Mater. 40 (1992) 1703-1716

[5] G.W. Greenwood, R.H. Johnson, Proc. R. Soc. Lond. 283A (1965) 403-422.

[6] P. Zwigl, D.C. Dunand, Metall. Mater. Trans. 29 (1998) 565-575.

[7] E. Gautier, A. Simon, G. Beck, Acta Metall. 35 (1987) 1367-1375.

[8] T.G. Nieh, J. Wadsworth, O.D. Sherby, Superplasticity in Metals and Ceramics, Cambridge, 1997.

[9] D.C. Dunand, in: T. Chandra, T. Sakai (Eds.), International Conference on Thermomechanical Processing of Steels and Other Materials, TMS, Warrendale, 1997, pp. 1821-1830.

[10] D.C. Dunand, S. Myojin, Mater. Sci. Eng. 230 (1997) 25-32.

[11] M. Frary, C. Schuh, D.C. Dunand, Metall. Mater. Trans. A 33 (2002) 1669-1680.

[12] U. Engel, R. Eckstein, J. Mater. Proc. Technol. 125 (2002) 35-44.

[13] Y. Saotome, H. Iwazaki, J. Mater. Proc. Technol. 119 (2001) 307-311.

[14] M. Zamora, J.P. Poirier, Mech. Mater. 2 (1983) 193-202.

[15] D.C. Dunand, C.M. Bedell, Acta Mater. 44 (1996) 1063-1076.

[16] H.J. Gonzalez, D.C. Dunand, J. Mater. Eng. Perform. 13 (2004) 665-669.

[17] C. Schuh, D.C. Dunand, J. Mater. Res. 16 (2001) 865-875.

[18] H.J. Frost, M.F. Ashby, Deformation-Mechanism Maps: The Plasticity and Creep of Metals and Ceramics, Pergamon Press, 1982.

[19] P. Zwigl, D.C. Dunand, Acta Mater. 45 (1997) 5285-5294.

[20] P. Zwigl, D.C. Dunand, Mater. Sci. Eng. 262 (1999) 166-172.

[21] C.A. Schuh, Philos. Magn. 82 (2002) 2441-2459.

[22] T. Nishihara, K. Asami, N. Iguchi, J. Jpn. Inst. Metals 41 (1977) 188-193.

[23] N. Iguchi, Y. Oka, Y. Saotome, J. Jpn. Inst. Metals 38 (1974) 725-730. 